Voix et Images

voixetimages

\title{
Entre identité et identitaire
}

\section{Shawn Huffman et Dominique Lafon}

Volume 33, numéro 1 (97), automne 2007

Michel Marc Bouchard

URI : https://id.erudit.org/iderudit/017523ar

DOI : https://doi.org/10.7202/017523ar

Aller au sommaire du numéro

Éditeur(s)

Université du Québec à Montréal

ISSN

0318-9201 (imprimé)

1705-933X (numérique)

Découvrir la revue

Citer cet article

Huffman, S. \& Lafon, D. (2007). Entre identité et identitaire. Voix et Images, 33(1), 9-14. https://doi.org/10.7202/017523ar d'utilisation que vous pouvez consulter en ligne.

https://apropos.erudit.org/fr/usagers/politique-dutilisation/ 


\title{
ENT R E I D E N T I T É ET I D ENT ITA I R E
}

\author{
SHAWN HUFFMAN \\ Université du Québec à Montréal \\ DOMINIQUE LAFON \\ Université d'Ottawa
}

Nous tenons à remercier très chaleureusement Michel Marc Bouchard de sa généreuse collaboration à l'élaboration de ce dossier.

En décrivant le thêâtre québécois des années 1980, on établit souvent une relation de cause à effet entre l'échec du premier référendum sur la souveraineté-association et l'émergence d'un thêâtre décrit comme étant plutôt intimiste ${ }^{1}$, apolitique ${ }^{2}$ et éclaté alors que la scène québécoise de la décennie précédente avait, surtout sur le plan poétique, pleinement participé aux aspirations d'indépendance du pays. Françoise Loranger et Robert Gurik, pour ne nommer que ceux-là, représentent une génération de dramaturges pour qui le théâtre est surtout un lieu de contestation politique et sociale. Il faut admettre que cet engagement contestataire est bien dans l'air du temps : le Living Theater à New York, les expériences theaâtrales issues de Mai '68 à Paris et le Thêâtre de l'opprimé à São Paulo exemplifient le rôle du théâtre dans la vie sociale et politique un peu partout dans le monde.

Comment comprendre ce qui arrive, du moins dans l'univers assez restreint du théâtre québécois, à la suite de l'échec du référendum ? S'agirait-il d'une remise en question collective de la notion même d'identité québécoise dont Michel Tremblay, dans ses pièces et dans ses entrevues, décrit souvent l'ambivalence, voire l'inauthenticité latentes et que le résultat du référendum aurait exacerbées? Ou faut-il plutôt interpréter ce ressac comme l'effet d'un traumatisme collectif provoqué par l'idée d'avoir perdu, peut-être à jamais, l'espoir d'une nation? Dans Cabaret neiges noires, Dominic Champagne reprend le titre de l'écrivain nationaliste Hubert Aquin pour mieux souligner une profonde aliénation vis-à-vis du récit unificateur nationaliste et en faire un cri de désillusion. Quoi qu'il en soit, le résultat

$$
+++
$$

1 Diane Pavlovic parle d'une "exploration des désordres privés». Voir "Le thêâtre québécois récent et l'américanité", Études françaises, vol. XXVI, nº 2, 1990, p. 42. 2 Madeleine Greffard et Jean-Guy Sabourin, par exemple, mettent en valeur des productions qui «défendent une forme apolitique misant sur le langage du corps et de la voix». Voir Madeleine Greffard et Jean-Guy Sabourin, Le thêâtre québécois, Montréal, Boréal, coll. «Boréal express», 1997, p. 110. 
du référendum force le récit nationaliste à céder la place à d'autres préoccupations et à d'autres esthétiques qui sont déjà en place, mais qui étaient jusque-là restées dans l'ombre. Le théâtre de Réjean Ducharme ou encore celui du Theâtre Expérimental de Montréal, fondé en 1975, illustrent ces premiers flirts avec la métathéâtralité, la discontinuité et le fragment qui font écho aux pratiques scéniques en émergence un peu partout en Europe et aux États-Unis et qui exerceront une influence indéniable sur la dramaturgie québécoise ${ }^{3}$. C'est dans ce contexte global de la postmodernité qu'émerge, au Québec, un theâtre qui participe de cette esthétique mondiale, mais qui aura ses propres couleurs. En 1980, la création presque simultanée de Panique à Longueuil, de René-Daniel Dubois, et de Rêve d'une nuit d'hôpital, de Normand Chaurette, suivie en 1981 par l'épopée Vie et mort du Roi Boiteux de Jean-Pierre Ronfard, marque le début de ce que l'on nommera «la nouvelle dramaturgie ${ }^{4}$ » caractérisée par une sorte de repli sur soi exprimé par toute une série d'interrogations autour de l'identité plutôt que de l'identitaire. C'est dans ce courant qu'émerge, en 1984, la production de Michel Marc Bouchard dont la famille, la sexualité et le rôle de l'individu-artiste constitueront les principaux lieux et sujets de réflexion.

Ce dossier est l'occasion, sinon d'une synthèse, du moins d'une relecture qui nous a permis de retracer les pistes d'une production touffue comme la forêt qui isole le Saguenay-Lac-Saint-Jean du reste du monde. Balisant quelques-uns des chemins parcourus par Michel Marc Bouchard, les titres de la quinzaine de pièces que comprend désormais le corpus bouchardien nous ont invités à nouer les fils d'une inspiration baroque qui conjugue le mythe, l'intime, le tragique et le mélodrame, la poésie et le calembour.

$\mathrm{Au}$ commencement étaient la fascination pour la tragédie grecque (Chrysippe), une certaine forme d'engagement social (écologiste) ainsi que le goût des couplages provocants, qui associent Chrysippe à Tanguay et la préservation de la nature au désir d'enfant d'un homosexuel. La contre-nature de Chrysippe Tanguay, pièce dont le titre fédérait déjà les composantes qui seront la marque de son thêâtre, a fait connaître Michel Marc Bouchard, qui avait alors à son actif quelques productions semi-professionnelles dont la liste, à elle seule, est révélatrice du dynamisme et de l'éclectisme du jeune auteur. Outre Scandale, pour laquelle il organise à quatorze ans une tournée dans sa région, on peut citer des pièces explicitement régionalistes comme Le retour inattendu de François Paradis et Les porteurs d'eau, et retenir Mortadelle, Angélus et dans Les bras de Morphée Tanguay. Inauguré dans cette production de jeunesse, le patronyme des Tanguay préfigure une généalogie de pièces familiales: La contre-nature de Chrysippe Tanguay, écologiste, puis La poupée de Pélopia, récemment remaniée ou plutôt réécrite sous le titre Des yeux de verre, Les muses orphelines et Soirée bénéfice pour tous ceux qui ne seront pas là en l'an 2000.

3 Jean-Cléo Godin et Dominique Lafon soulignent, par exemple, l'apport du thêâtre allemand, évoquant même une "Allemagne québécoise» pour qualifier cette influence directe. Voir Dramaturgies québécoises des années quatre-vingt, Montréal, Leméac, coll. «Thêâtre/Essai», 1999, p. 27. 4 L'expression est proposée par JeanCléo Godin dans «Deux dramaturges de l'avenir?», Études thêâtrales, vol. XVIII, n 3, 1985, p. 113. 
Bien qu'elles n'établissent aucun lien familial entre leurs personnages respectifs ${ }^{5}$, ces pièces déclinent les figures d'un même topos, celui de la conception. Au désir d'enfant de Chrysippe, dont la demande d'adoption avortera (au sens concret ou peu s'en faut ${ }^{6}$ ) au terme de l'enquête menée par une agente d'adoption, succédera le désir de son propre enfant, désir incestueux d'un créateur de poupées pour la fille qui lui a servi de modèle. Ces deux théâtralisations de la gestation paternelle, à des titres divers "contre-nature ", qui exacerbaient certains enjeux sociologiques (la parentalité homosexuelle, l'inceste paternel), seront suivies de deux conceptions maternelles, également problématiques. Dans Les muses orphelines, c'est l'absence de la mère qui est remise en question par sa mise en scène, qu'elle soit mimée par le fils, caricaturée par la sœur aînée ou réinventée par la benjamine dans l'annonciation finale d'une providentielle grossesse. Bien qu'elle laisse dans l'ombre son avenir, cette conception libère le personnage de l'enfer familial et demeure à ce jour la seule note optimiste et féminine de l'œuvre. Soirée bénéfice pour tous ceux qui ne seront pas là en l'an 2000, quelques années plus tard, donnera à l'enfer familial son apocalypse et son éternité. Nés de l'union «contrenature» (et contre tout réalisme) d'un loup et d'une femme, les derniers Tanguay, stériles et immortels, mettront à mal leur mère, celle qui les rattachait au vrai monde par le négoce de l'entreprise de fourrures familiales. Les Tanguay étaient entrés dans la légende et le cycle s'acheva dans l'étrange fin du monde millénariste d'une pièce qui n'a jamais été publiée, mais dont on trouvera ici quelques extraits.

Cette pièce marqua aussi la fin des filiations: les patronymes disparurent de l'œuvre même si les liens familiaux demeuraient au cœur de sa dynamique conflictuelle. Le meurtre succèda à la gestation. Les fils du Chemin des passes-dangereuses ne portent pas le nom de leur père, auquel la pièce n'attribue aucune fonction sociale, si ce n'est, dérisoire, un talent poétique qui doit beaucoup à l'éthylisme... De façon très significative, ils sont, comme les lycanthropes Tanguay, en panne, au bord de la route, unis dans la mort alors que leur mode de vie respectif les avait éloignés, au propre et au figuré, les uns aux autres. La fratrie n'est plus une meute, mais le lieu d'une différenciation que souligne le dispositif familial du Voyage du couronnement. Le père, auquel on ne s'adresse qu'en utilisant son surnom, «Le Caïd», a deux fils. Ce n'est que dans la liste des personnages qu'ils partagent un même patronyme, Bérubé, alors que leurs prénoms (Hyacinthe et Sandro), qui leur ont été donnés par deux mères respectivement d'origine française et italienne, les distingueront tout au long de la pièce. Le but du voyage du Caïd est de changer de vie et de changer de nom de famille. Les Bérubé doivent, au terme de la traversée, devenir les Peacock. Le Caïd effacera ainsi son dossier criminel; ses deux fils, désormais Martin et James, leur différence. On mesure ici le rôle névralgique joué par l'onomastique dans une pièce dont l'enjeu politique est explicite non seulement dans le titre, mais aussi au sein du groupe de trois personnages secondaires, toutes

5 Le nom de famille des premières versions de Soirée bénéfice est Mercier. Sa filiation patronymique avec les autres pièces est donc en quelque sorte «factice». 6 Chrysippe, au dénouement, annonce: "Je suis très malade, j'ai fait une fausse-couche.» 
lauréates d'un concours auquel ne participaient que des Élisabeth 7 . Dans Les manuscrits du déluge, les protagonistes ne sont désignés que par des prénoms alors qu'ils déclinent, à la manière des belles-sœurs de Tremblay, la liste des patronymes du village. Dans le nom du personnage se joue ainsi son existence, mais aussi son essence. Sous le regard des mouches le montre bien qui ignore le patronyme familial, mais change les prénoms des amoureux par l'antonomase ${ }^{8}$ (Docile) ou par la mise en abyme du nom d'un héros de roman (Peter). Tout se passe comme si, au fil de l'œuvre (un peu comme chez Tremblay), l'identité des personnages devait s'estomper pour mieux laisser voir la caractérisation symbolique que leur attribue un dramaturge qui, de surcroît, renvoie de plus en plus explicitement, par le jeu des références toponymiques ou par les confidences des préfaces, à des données plus personnelles.

C'est avec Les feluettes que le dramaturge connaît la gloire et la reconnaissance internationale, mais c'est aussi avec cette pièce que le terroir dont il est issu accède au statut de lieu mythique. Le Lac-Saint-Jean y devient le creuset dans lequel fusionnent les éléments qui caractérisent et expliquent les relations entre les personnages. L'aérienne Lydie-Anne ne descend de son aérostat que pour enflammer le désir de Simon l'incendiaire; le fluide Vallier, le feluette, trouve chez sa mère, la Comtesse qui se nourrit de terre et d'humus, le courage de se tenir droit et d'avouer, à la face du monde son amour pour Simon. La revendication amoureuse du couple homosexuel, sublimée par la rencontre symbolique de l'eau et du feu, accède ainsi à une évanescence alchimique qui déjoue les préjugés dont il est trop souvent l'objet. Les manuscrits du déluge, comme Le peintre des madones rediront la connaissance intime, l'amour de l'auteur pour cette région que les deux pièces dépeignent respectivement comme une arche de Noé ou comme le lieu d'un sabbat de sorcières. La référence personnelle, qui se lisait dans la composition de la famille des Muses orphelines ou dans le rappel de la boucherie familiale dans Sous le regard des mouches, participe désormais d'un registre mythologique dont l'auteur se ferait l'aède. Dans l'entretien qui figure dans le présent dossier, Michel Marc Bouchard ne confie-t-il pas que, pour lui, sa région recèle «dans chaque village, assez de matière pour une représentation plus symbolique, plus mythique même» du réel?

Le thêâtre d'été de Michel Marc Bouchard, et en particulier Les grandes chaleurs et Les papillons de nuit qui ont connu un grand succès au sein de ce répertoire, réitère dans le registre comique la mise en question de l'univers familial en s'autorisant quelques audaces qui allient métaphore scénique et symbolisme. Dans la première pièce, les cendres du défunt père sont dans un sac d'aspirateur, mais le récit des circonstances entourant leur hasardeuse dispersion s'accompagne de révélations concernant la sexualité de la mère et l'homosexualité du fils. Et que dire, dans la seconde, de l'analphabétisme d'une mère obsédée par la propreté et qui oblige ses enfants à épeler encore une fois les non-dits d'une saga familiale en quête

7 Tous les personnages féminins du Peintre des madones porteront des prénoms composés à partir de Marie....

8 Les membres du dérisoire groupe rock de Rock pour un faux bourdon (1983), qui pratiquent le lip-synch, étaient désignés par des surnoms qui, tels des totems scouts, assimilaient les meneurs à des insectes ou préfiguraient, en d'ironiques antonomases telles Popstar, la faiblesse de leurs comparses. 
de substitut paternel? La blessure qui hante l'œuvre de Michel Marc Bouchard se cache aussi dans le rire.

Nous nous étions fixé plusieurs objectifs lors de la préparation de ce dossier: aborder le parcours artistique du dramaturge; réunir une série d'études représentatives des différentes périodes de sa production - du "cycle Tanguay" jusqu'aux pièces les plus récentes; privilégier les sujets moins étudiés dans le corpus critique: la violence, l'importance de la mémoire, la religion et le traumatisme, entre autres. Or un fil conducteur traverse tout le dossier: celui de la blessure. Celle dont l'auteur parle dans l'entretien, à savoir cette blessure fondamentale qui serait commune à tous les écrivains, mais aussi celles qu'il ne fait qu'évoquer ou dont il refuse carrément de parler. Ce sont ces blessures que les articles et les documents de ce dossier - l'entretien et l'inédit - auscultent dans le corp(u)s bouchardien.

Dans son étude, Lucie Robert se penche sur la figure de l'artiste - écrivains, peintres, musiciens - dans l'œuvre de Michel Marc Bouchard et dans la tragédie grecque classique. Elle s'attache plus particulièrement à l'analyse d'une image récurrente à l'intérieur de cette figure, à savoir la blessure qui habite l'artiste et l'empêche d'accéder à la création. Or cette stérilité artistique - comme le souligne Robert, l'artiste bouchardien confond souvent création et procréation - est rarement surmontée. Dominique Lafon, quant à elle, interroge plutôt la blessure qui émerge de la violence ou dont la violence est un symptôme. S'appuyant sur les travaux de René Girard, elle cherche à en identifier les fonctions et les sphères d'action et conclut à la possibilité de l'existence, dans l'œuvre, d'un registre de la violence plus élargi, qui s'étendrait même au-delà de la famille, microcosme de la socialité chez Bouchard, et dont le fonctionnement reposerait sur un principe d'inversion des mythes fondateurs.

En prenant comme point de départ une blessure mortelle, celle de l'accident de route dans Le chemin des passes-dangereuses, Mariel O'Neill-Karch analyse la blessure familiale et identitaire dont souffrent les trois protagonistes jusque dans leur agonie. S'inspirant de la notion d'«impression», empruntée à Baudrillard, O’Neill-Karch identifie une sorte de «défaillance d'être» dans cette pièce. S'il s'agit d'une «impression» d'être - les trois frères sont déjà morts - il reste que ces personnages ont également été marqués dans leur être par la honte, la culpabilité et la colère. Pour sa part, Shawn Huffman aborde une autre forme de blessure récurrente dans la dramaturgie de Michel Marc Bouchard: celle de la violence physique, plus particulièrement la violence faite aux enfants. En effet, le dramaturge met souvent en scène des univers où l'adulte fait un retour sur l'espace de son enfance pour mieux comprendre son passé. Il en va ainsi dans L'histoire de l'oie, l'une des pièces les plus célèbres du dramaturge, dans laquelle l'espace et le mythe contribuent à créer une figure de traumatisme qui est mue par la violence physique. Cette figure se transforme toutefois au fur et à mesure que le protagoniste s'approprie son passé, ce qui lui permet en outre de trouver une issue à l'impasse dans laquelle se trouve sa vie.

C'est donc une image assez sombre que pourrait laisser au terme de sa traversée, une œuvre hantée par la souffrance des corps, les affres de la création, les blessures de l'enfance. Mais ce serait ne pas prendre en compte la dimension 
formelle de l'œuvre qui abolit, dans l'humour grinçant ou la dérision de certaines thématiques convenues, les frontières des genres canoniques. Ce serait également oublier qu'elle se joue des préjugés dont sont victimes les homosexuels, qu'elle dénonce la solitude dans laquelle le règne du jeunisme relègue les personnes âgées. Oublier surtout que tout en portant un regard critique sur la société québécoise et son histoire, elle n'en élève pas moins le terroir, le pays, au rang des mythes fondateurs et qu'elle tente de réaliser, du même coup, la symbiose de l'identité et de l'identitaire. 